\title{
Psychosis Following Anti-Obesity Treatment with Rimonabant
}

\author{
Tarik Ugur $^{\mathrm{a}} \quad$ Marius Bartels $^{\mathrm{a}} \quad$ Bernhard Kis $^{\mathrm{a}} \quad$ Norbert Scherbaum $^{\mathrm{b}}$ \\ a Department of Psychiatry and Psychotherapy, \\ b Department of Addictive Behavior and Addiction Medicine, Rhine State Hospital, University of Duisburg-Essen, Germany
}

\section{Key Words}

Rimonabant · Obesity · Cannaboid · Schizophrenia · Antipsychotic

\section{Summary}

Background: In this report, we present the case of a patient with a relapse of schizophrenia following an episode of depression and increased anxiety after antiobesity treatment with rimonabant, a cannabinoid type 1 receptor antagonist. Case Report: After 4 weeks of treatment the patient developed psychiatric symptoms, i.e. depressed mood and elevated anxiety. Four months after the discontinuation of rimonabant, the patient presented with psychotic symptoms fulfilling ICD-10 criteria of paranoid schizophrenia. Antipsychotic treatment with quetiapine was initialized. A stable recovery took further 4 weeks in which combined treatment with quetiapine and ziprasidone was given. Conclusion: The course of the illness suggests that the continuous affective symptoms, which were most likely a side effect of rimonabant, may have triggered the psychosis analogous to the stress-diathesis model of schizophrenia. As a consequence, rimonabant may not be the first choice in obese patients with a history of schizophrenia due to a potentially increased risk of a relapse via an indirect mechanism.

\section{Introduction}

Schizophrenia is a chronic and relapsing illness that impairs mental and social functioning. Its phenomenology includes a variety of symptoms ranging from positive symptoms such as delusions and hallucinations to negative symptoms such as avolition, anhedonia or alogia. Atypical antipsychotics are effective medications of positive and negative symptoms. However, a considerable number of patients develop side effects. Weight gain is one of the most common side effects and a major factor for the discontinuation of the medication. Furthermore, a clinical dilemma arises when the treating psychiatrist needs to consider the health risks posed by obesity on the one hand and the risk of a relapse on the other hand.

In recent years cannabinoid receptor antagonists have been proposed to treat a variety of conditions, ranging from nicotine addiction [1] to obesity [2,3]. Rimonabant is a typical representative of this emerging class and has been approved by the European Medicines Evaluation Agency (EMEA) in 2006 as an anorectic anti-obesity drug. It is indicated for use in conjunction with diet and exercise for patients with a BMI greater than $30 \mathrm{~kg} / \mathrm{m}^{2}$ or for patients with a BMI greater than $27 \mathrm{~kg} / \mathrm{m}^{2}$ with associated cardiovascular risk factors. However, the advisory panel of the US Food and Drug Administration (FDA) has rejected the sale of rimonabant in the USA in June 2007 due to concerns that it potentially causes neurological and psychiatric problems including an increased risk of suicide. Therefore, the FDA is not expected to approve rimonabant as an anti-obesity drug within the USA without additional safety data.

Pharmacologically rimonabant is a cannabinoid type $1\left(\mathrm{CB}_{1}\right)$ receptor antagonist. Together with lipid-derived ligands the $\mathrm{CB}_{1}$ receptors are an integral part of the endocannabinoid system [4]. They are expressed both inside and outside the CNS [5]. They are present in the olfactory bulbs, some cortical regions, and several subcortical regions such as some parts of basal ganglia, thalamic and hypothalamic nuclei. Outside the CNS CB1 receptors are mainly found in adipose tissue, muscle cells, and digestive organs. The endocannabinoid system seems to modulate glucose and lipid metabolism $[6,7]$. There is

\begin{tabular}{|c|c|}
\hline KARGER & (C) 2008 S. Karger GmbH, Freiburg \\
\hline $\begin{array}{l}\text { Fax +49 } 7614520714 \\
\text { E-mail Information@Karger.de } \\
\text { www.karger.com }\end{array}$ & $\begin{array}{l}\text { Accessible online at: } \\
\text { www.karger.com/ofa }\end{array}$ \\
\hline
\end{tabular}

Dr. med. Tarik Ugur

Abteilung für Psychiatrie und Psychotherapie

Rheinische Kliniken Essen, Universität Duisburg-Essen

Virchowstraße 174, 45147 Essen, Germany

Tel. +49 201 40860-111, Fax -151

E-mail Tarik.Ugur@uni-duisburg-essen.de 
mounting evidence that it is inadequately activated in obesity [8]. An agonistic modulation of the cerebral $\mathrm{CB}_{1}$ receptors as is the case with tetrahydrocannabinol (THC) results in subjective effects such as euphoria, marked distortions in perception, psychotic phenomena, and also an appetite-enhancing effect. The psychotogenic effects are epidemiologically well established and may be due to the fact that agonists such as THC increase dopamine release [9]. Since rimonabant has an antagonistic impact on $\mathrm{CB}_{1}$ receptors, it decreases appetite [2] and may have antipsychotic propensities due to a decrease of the mesolimbic dopamine release [10]. However, a first trial of this hypothesis was not conclusive [11].

\section{Case Report}

Here we present the case of a 37-year-old woman with no family history of psychosis or any other psychiatric illness. Her history revealed that she was diagnosed with schizophrenia at the age of 29. During the early course of her illness, she was treated with oral antipsychotics on an outpatient basis. Her early professional life was interrupted by one psychotic episode which made an inpatient treatment necessary. Since she performed well in the following years, her medication was reduced to $200 \mathrm{mg}$ amisulpride daily. She remained stable over the following 5 years. Nevertheless, she noticeably gained weight, with a BMI increase from 22 to $29 \mathrm{~kg} / \mathrm{m}^{2}$; her weight fluctuated around $85 \mathrm{~kg}$ at a height of $173 \mathrm{~cm}$. The patient undertook efforts to avoid further weight gain by regulating dietary intake, exercise and participating in a commercial diet program which failed. Her general practitioner then prescribed rimonabant (Acomplia ${ }^{\circledR}$; Sanofi-Aventis, Frankfurt/M., Germany) $20 \mathrm{mg}$ daily, which had just become available. There was no evidence for an uncontrolled serious psychiatric illness at the time of prescription. No data regarding mental status, weight, blood pressure, glucose and lipids during the treatment were retrospectively available.

According to the patient, she developed a depressed mood and sleep problems with frequent nightmares within 4 weeks of treatment. During the following weeks, she experienced nightmares as through driven by external forces and suffered from occasional visual hallucinations mostly in the form of bright spots and halo phenomena. In a desperate attempt to alleviate her depression she tried St. John's wort as a self-medication with little effect. After a total of 4 months the patient considered that rimonabant may be the cause of her deteriorating psychiatric condition and stopped the medication. Following a brief initial relief her delusions intensified, and she experienced auditory hallucinations for the first time in her life. Her admission into our psychiatric ward followed an advice by a neurologist she had consulted for vertigo.

On admission (4 months after discontinuation of the medication), psychiatric examination revealed a middle-aged woman who was able to maintain eye contact and rapport but appeared perplexed. Her train of thoughts was tangential and sometimes slightly incoherent. There was evidence of persecutory delusions. The patient complained of hearing thoughts spoken aloud, reported low mood, and had a slightly inappropriate affect. She did not experience suicidal thoughts. Sleep and appetite were impaired. The neurological assessment was unremarkable, with no evidence of focal neurological signs, movement disorder, or soft neurological signs. Investigations included routine blood tests, which were within normal ranges, and an electrocardiogram, which was also normal. The symptoms matched criteria for paranoid schizophrenia (F20.0) according to the International Classification of Diseases (ICD-10) [12].

The patient was given quetiapine resulting in a marked improvement of positive symptoms after 3 weeks of treatment. Following an initial response to treatment, the patient complained about intolerable side effects of weight gain and drowsiness; two other atypical antipsychotics were subsequently prescribed. A lasting recovery was maintained under ziprasidone with a weekly reduction of quetiapine by $10 \%$.

Informed written consent was obtained prior to publication 2 months after recovery.

\section{Discussion}

Several studies assessed the efficacy of rimonabant in overweight or obese patients. The primary aims of these trials included the assessment of weight reduction and the reduction of cardiovascular risk factors such as hypertension, type 2 diabetes and dyslipidemia [13-15]. Patients with a clinically significant psychiatric disorder or who were currently using antidepressants were excluded. Nevertheless, psychiatric disorders were relatively common adverse effects during the trials. Their incidence exceeded 5\%. Psychiatric symptoms, including depressed mood, anxiety and aggression, were also a major cause for study discontinuation. No other serious psychiatric disorders or other serious adverse events linked to psychiatric disorders were recorded during the trials. Currently, no safety data pertaining to the use of rimonabant with psychosis-prone subjects are available. According to the product characteristics of Acomplia [16], any ongoing major depressive illness and antidepressive treatment are contraindications for the use of rimonabant. Furthermore, therapy with rimonabant is not recommended in patients with uncontrolled serious psychiatric illness. If patients with a history of mood alterations are considered for treatment, the manufacturer advises a careful risk evaluation and a monitoring for the emergence of affective symptoms.

In the case presented here, there was no evidence of depression or uncontrolled psychiatric illness at the time when rimonabant was prescribed. With respect to the history of mood alterations before and during psychotic episodes, it could be discussed whether the treatment was contraindicated. In any case, the use of rimonabant is not recommended in patients with $\mathrm{BMI}<30 \mathrm{~kg} / \mathrm{m}^{2}$ without medical sequelae of obesity; the patient had had a BMI of $29 \mathrm{~kg} / \mathrm{m}^{2}$ upon initiation of rimonabant treatment. Treatment should have been stopped after the affective symptoms emerged. Given the previously unsuccessful diets, the treating physician who was not a specialist in psychiatry opted for an off-label use. The situation was further complicated when regular consultations as recommended by the manufacturer were missed by the patient due to her deteriorating mental status. Hence, initial symptoms in the form of mild depression and elevated anxiety were not noticed during treatment. Minimal psychotic symptoms also appeared at an early stage. A full-blown psychosic episode developed with a latency of a few months following the discontinuation of rimonabant and despite being on antipsychotic treatment.

These clinical observations contradict theoretical considerations that cannabinoid receptor antagonists unfold antipsychotic effects $[10,11]$. These theoretical considerations are 
widely supported by findings in rodents, which suggest a negative impact of rimonabant on dopamine D2 receptor-mediated neurotransmission [15] as well as functional and metabolic similarities between antipsychotics and rimonabant $[16,17]$. Therefore, we discuss the possibility of a spontaneous relapse which may have merely coincided with the anti-obesity treatment. Spontaneous relapses during the natural course of schizophrenia are common and empirically well established. However, the illness had a relatively benign course, and a remission had been maintained for half a decade. Regarding the psychiatric history of this patient, a combination of triggering factors for the relapse has to be considered. Depression and anxiety are known side effects of rimonabant. At the same time they are major stressors for patients with a history of psychosis. We postulate that the preceding depression triggered the relapse, according to the stress-diathesis model [19-21]. With respect to the above mentioned similarities between antipsychotics and rimonabant, we hypothesize that an antagonistic stimulation of limbic $\mathrm{CB}_{1}$ receptors may have caused the depressive symptoms through a decrease in dopamine release in core structures of the cerebral reward pathway. An analogous model has already been proposed for antipsychotics [22]. It is yet unknown whether the antidopaminergic effects of antipsychotics and cannabinoid receptor antagonists are additive or whether they unfold some kind of drug synergism. Further research is needed to verify the correlation between an antagonistic stimulation of the endocannabinoid system and schizophrenia as well as the underlying mechanism. Our case report suggests that rimonabant can indirectly induce acute psychosis in patients with a history of schizophrenia.

\section{References}

1 Cahill K, Ussher M: Cannabinoid type 1 receptor antagonists (rimonabant) for smoking cessation. Cochrane Database Syst Rev 2007;17:CD005353.

2 Kirkham TC, Tucci SA: Endocannabinoids in appetite control and the treatment of obesity. CNS Neurol Disord Drug Targets 2006;5:272-92.

3 Curioni C, André C: Rimonabant for overweight or obesity. Cochrane Database Syst Rev 2006;18:CD 006162. DOI: $10.1002 / 14651858$

$\checkmark 4$ Howlett AC, Breivogel CS, Childers SR, Deadwyler SA, Hampson RE, Porrino LJ: Cannabinoid physiology and pharmacology: 30 years of progress. Neuropharmacology 2004;47:345-58.

$\checkmark 5$ Matsuda LA, Lolait SJ, Brownstein MJ, Young AC, Bonner TI: Structure of a cannabinoid receptor and functional expression of the cloned cDNA. Nature 1990;346:561-4.

6 Di Marzo V, Goparaju SK, Wang L, Liu J, Bátkai S, Járai Z, Fezza F, Miura GI, Palmiter RD, Sugiura T, Kunos G: Leptin-regulated endocannabinoids are involved in maintaining food intake. Nature 2001; 410:822-5.

7 Poirier B, Bidouard JP, Cadrouvele C, Marniquet X Staels B, O'Connor SE, Janiak P, Herbert JM: The anti-obesity effect of rimonabant is associated with an improved serum lipid profile. Diabetes Obes Metab 2005;7:65-72.

8 Engeli S: Peripheral metabolic effects of endocannabinoids and cannabinoid receptor blockade. Obesity Facts 2008;1:8-15.

9 Gardner EL: Endocannabinoid signaling system and brain reward: emphasis on dopamine. Pharmacol Biochem Behav 2005;81:263-84.
10 Ballmaier M, Bortolato M, Rizzetti C, Zoli M, Gessa G, Heinz A, Spano P: Cannabinoid receptor antagonists counteract sensorimotor gating deficits in the phencyclidine model of psychosis. Neuropsychopharmacology 2007;32:2098-107.

11 Meltzer HY, Arvanitis L, Bauer D, Rein W, for the Meta-Trial Study Group: Placebo-controlled evaluation of four novel compounds for the treatment of schizophrenia and schizoaffective disorder. Am J Psychiatry 2004;161:975-84.

12 Pi-Sunyer FX, Aronne LJ, Heshmati HM, Devin J, Rosenstock J, for the RIO-North America Study Group: Effect of rimonabant, a cannabinoid-1 receptor blocker, on weight and cardiometabolic risk factors in overweight or obese patients: RIO-North America: a randomized controlled trial. JAMA 2006;295:761-75.

13 Van Gaal LF, Rissanen AM, Scheen AJ, Ziegler O, Rössner S, for the RIO-Europe Study Group: Effects of the cannabinoid-1 receptor blocker rimonabant on weight reduction and cardiovascular risk factors in overweight patients: 1-year experience from the RIO-Europe study. Lancet 2005;365: 1389-97.

14 Scheen AJ, Finer N, Hollander P, Jensen MD, Van Gaal LF; RIO-Diabetes Study Group: Efficacy and tolerability of rimonabant in overweight or obese patients with type 2 diabetes: a randomised controlled study. Lancet 2006;368:1660-72.

15 Melis T, Succu S, Sanna F, Boi A, Argiolas A, Melis MR: The cannabinoid antagonist SR 141716A (rimonabant) reduces the increase of extra-cellular dopamine release in the rat nucleus accumbens induced by a novel high palatable food. Neurosci Lett 2007;419:231-5.
16 Acomplia: Summary of Product Characteristics. Sanofi Aventis, 2007.

17 Nagai H, Egashira N, Sano K, Ogata A, Mizuki A, Mishima K, Iwasaki K, Shoymam Y, Nishimura R, Fujiwara M: Antipsychotics improve delta9-tetrahydrocannabinol-induced impairment of the prepulse inhibition of the startle reflex in mice. Pharmacol Biochem Behav 2006;84:330-6.

18 Alonso R, Voutsinos B, Fournier M, Labie C, Steinberg R, Souilhac J, Le Fur G, Soubrié P: Blockade of cannabinoid receptors by SR141716 selectively increases Fos expression in rat mesocorticolimbic areas via reduced dopamine D2 function. Neuroscience 1999;91:607-20.

19 Zubin J, Spring B: Vulnerability - a new view of schizophrenia. J Abnorm Psychol 1977;2:103-24.

20 Day R, Nielsen JA, Korten A, Ernberg G, Dube KC, Gebhart J, Jablensky A, Leon C, Marsella A, Olatawura M, et al: Stressful life events preceding the acute onset of schizophrenia: a cross-national study from the World Health Organization. Culture Med Psychiatry 1987;11:123-205.

21 Corcoran C, Walker E, Huot R, Mittal V, Tessner K, Kestler L, Malaspina D: The stress cascade and schizophrenia: etiology and onset. Schizophr Bull 2003;29:671-92.

22 Kirsch P, Ronshausen S, Mier D, Gallhofer B: The influence of antipsychotic treatment on brain reward system reactivity in schizophrenia patients. Pharmacopsychiatry 2007;40:196-8. 